\title{
GASOMETRÍA ARTERIAL EN ADULTOS CLÍNICAMENTE SANOS A 3350 METROS DE ALTITUD
}

\author{
César J. Pereira-Victorio ${ }^{1,2, a, b}$, John Huamanquispe-Quintana ${ }^{3,4, a}$, \\ Luis E. Castelo-Tamayo $0^{5,6, \mathrm{c}}$
}

\begin{abstract}
RESUMEN
Objetivo. Determinar los valores gasométricos arteriales de normalidad, en adultos clínicamente sanos que viven a 3350 metros de altitud. Materiales y métodos. Estudio realizado en Cusco, distrito de Santiago a $3350 \mathrm{~m}$ de altitud, se determinó una muestra de 118 sujetos mediante un estudio piloto previo, seleccionados por muestreo por conveniencia; las personas tenían entre 20-59 años de edad, sin antecedentes patológicos pulmonares, cardiovasculares o hematológicos, con práctica deportiva menor a $60 \mathrm{~min} / \mathrm{día}$, sin hábito tabáquico, residentes los 5 años previos al estudio en Cusco y catalogados como "clínicamente sanos" por dos médicos internistas y un neumólogo. La recolección gasométrica fue estandarizada, para el análisis estadístico se empleó medidas de tendencia central y dispersión, t de Student y análisis de varianza, correlación lineal y regresión lineal múltiple. Resultados. Las mujeres conformaron el 57,6\% de la muestra; la frecuencia respiratoria fue 16,2 por minuto, y el IMC 24,8. Los resultados gasométricos fueron: $\mathrm{pH}=7,42 \mathrm{mEq} / \mathrm{L}$; $\mathrm{pO}_{2}=61,08 \mathrm{mmHg} ; \mathrm{pCO}_{2}=30,62 \mathrm{mmHg} ; \mathrm{pAO}_{2}=62,52 \mathrm{mmHg} ; \mathrm{SO}_{2}=91,13 \% ; \mathrm{AaDO}_{2}=0,0 \mathrm{mmHg} ; \mathrm{Hto} 44,22 \% ; \mathrm{Hb} 14,74 \mathrm{mg} /$ dL; CaO2 18,18 vols/\%; $\mathrm{HCO} 3$ 19,74mmol/L; $\mathrm{pO}_{2} / \mathrm{FiO}_{2} 290,79$ y Anion Gap 20,99. Mediante regresión lineal, a partir de los 20 años de edad, por cada año cumplido, "disminuyen" $\mathrm{pO}_{2}(0,122 \mathrm{mmHg}), \mathrm{pAO}_{2}(0,08 \mathrm{mmHg}), \mathrm{SO}_{2}(0,05 \%)$, índice $\mathrm{pO}_{2} / \mathrm{FiO}_{2}(0,571 \mathrm{mmHg})$; e "incrementa" la pCO $(0,056 \mathrm{mmHg})$. Conclusiones. Los resultados hallados respecto a los parámetros gasométricos son diferentes a los del nivel de mar y pueden ser empleados en poblaciones que habitan a altitud semejante a la del estudio.
\end{abstract}

Palabras clave: Análisis de los gases de la sangre; Altitud; Hipoxia (fuente: DeCS BIREME).

\section{ARTERIAL BLOOD GASES IN CLINICALLY HEALTHY ADULTS LIVING AT 3,350 METERS OF ALTITUDE}

\begin{abstract}
Objectives. To determine the normal values of arterial blood gases in clinically healthy adults living at 3,350 meters of altitude. Materials and methods. The study was conducted in the Santiago district of Cusco, Peru at 3,350 meters of altitude with a sample of 118 subjects determined in a previous pilot study and selected by convenience sampling. The sample included individuals between 20-59 years old with no pulmonary, cardiovascular and hematological medical history, who practice sports less than 60 minutes per day, non smoking, have resided at least 5 years prior to the study in Cusco and were characterized through clinical examination by two internists and pulmonologists as "clinically healthy". The arterial blood gas collection was standardized for statistical analysis measures of central tendency and dispersion. Student's t-tests and analysis of variance, linear correlation and multiple linear regression were applied. Results. Women comprised $57.6 \%$ of the sample. Respiratory rate averaged 16.2 per minute and BMI 24.8. The blood gas results were: pH $7.42 \mathrm{mEq} / \mathrm{L} ; \mathrm{pO}_{2} 61.08 \mathrm{~mm} \mathrm{Hg} ; \mathrm{pCO}_{2} 30.62 \mathrm{~mm} \mathrm{Hg} ; \mathrm{pAO}_{2} 62.52 \mathrm{~mm} \mathrm{Hg} ; \mathrm{SO}_{2}$ 91.13\%; $\mathrm{AaDO}_{2} 0.0 \mathrm{~mm} \mathrm{Hg}$; Hto 44.22\%; $\mathrm{Hb} 14.74 \mathrm{mg} / \mathrm{dL}$; CaO2 18.18 vol\%; $\mathrm{HCO} 319.74 \mathrm{mmol} / \mathrm{L} ; \mathrm{pO}_{2} / \mathrm{FiO}_{2} 290.79$ and Anion Gap 20.99. Using linear regression, it was observed that each year from the age of 20 , there was a decrease in $\mathrm{pO}_{2}(0.122 \mathrm{~mm} \mathrm{Hg}), \mathrm{pAO}_{2}(0.08$ $\mathrm{mm} \mathrm{Hg}), \mathrm{SO}_{2}(0.05 \%)$, index $\mathrm{pO}_{2} / \mathrm{FiO}_{2}(0.571 \mathrm{~mm} \mathrm{Hg})$ and an increase in $\mathrm{pCO}_{2}(0.056 \mathrm{~mm} \mathrm{Hg})$. Conclusions. The results found with respect to blood gases in this population are different from those residing at sea level and can be used to study other populations residing at a similar altitude.
\end{abstract}

Key words: Blood gas analysis; Altitude; Hypoxia (source: MeSH NLM).

\footnotetext{
Complejo Hospitalario Universitario de Albacete. Albacete, España.

Universidad Complutense de Madrid. Madrid, España.

Universidad Nacional de San Antonio Abad, Cusco, Perú.

Hospital Nacional Adolfo Guevara Velasco. Cusco, Perú.

Universidad Andina del Cusco. Cusco, Perú.

Hospital Antonio Lorena del Cusco. Cusco, Perú.

Médico cirujano; ${ }^{\mathrm{b}}$ máster en salud pública; ${ }^{\mathrm{c}}$ médico internista

Recibido: 28-01-14 Aprobado: 06-08-14
}

Citar como: Pereira-Victorio CJ, Huamanquispe-Quintana J, Castelo-Tamayo LE. Gasometría arterial en adultos clínicamente sanos a 3350 metros de altitud. Rev Peru Med Exp Salud Publica. 2014;31(3):473-79. 


\section{INTRODUCCIÓN}

Las condiciones de hábitat a nivel del mar difieren de las presentes en zonas montañosas, siendo más notoria la diferencia a partir de una altura moderada de 2450 metros sobre el nivel del mar (1). La altitud connota variación de condiciones a las que se debe adaptar el ser vivo; para las personas que viven a nivel del mar, los ascensos a ciudades situadas a más de $2400 \mathrm{~m}$ de altitud pueden desencadenar problemas como el "mal de montaña agudo"; en cambio, personas nativas y residentes en ciudades de mayor altitud a la citada, pueden realizar con normalidad sus actividades, esto es debido a las características propias de la relación presión atmosférica-altitud y al fenómeno biológico de adaptación que todos los seres vivos presentamos (2). Existen otros factores indirectos que tienen un papel importante y están condicionados por la misma altitud, la temperatura del aire disminuye aproximadamente $1{ }^{\circ} \mathrm{C}$ por cada $150 \mathrm{~m}$ de elevación y la humedad ambiental es menor proporcionalmente a la disminución de la temperatura ${ }^{(2)}$.

Los fenómenos de adaptación a la altitud podrían explicar las diferencias morfológicas y fisiológicas que existen entre el hombre alto-andino respecto a los que viven a nivel del mar, por ejemplo: la circunferencia torácica es superior en un 12 a $15 \%$ y se incrementa a mayor altitud y tiempo de residencia condicionando mayores volúmenes y capacidades pulmonares ${ }^{(3,4)}$, la adaptabilidad en los primeros años de vida es importante en la determinación de la función pulmonar ${ }^{(5)}$. Se produce una disminución en la presión alveolar de oxígeno $\left(\mathrm{pAO}_{2}\right)$ con estimulación refleja de hiperventilación, por encima de los $4500 \mathrm{~m}$, la ventilación es $20 \%$ mayor y hasta $40 \%$ si es ajustada por talla, esta hiperventilación se ve atenuada proporcionalmente según el tiempo de vida en altitud ${ }^{(6-9)}$. Respecto al dióxido de carbono $\left(\mathrm{CO}_{2}\right)$, la sensibilidad es menor debida a la hipoxia, no hay alteración de los quimiorreceptores periféricos y el tiempo de respuesta es más lento ${ }^{(10,11)}$.

Presentan hiperplasia en la medula ósea, con mayor porcentaje de eritrocitos que granulocitos; los eritrocitos tienen más tiempo de vida y mayor recambio de hierro (12); la tasa de producción de glóbulos rojos puede aumentar hasta diez veces luego de un ascenso notorio de eritropoyetina (EPO), incremento de producción que se mantiene, incluso, cuando los niveles de EPO se normalizan (13); la diferencia de hemoglobina $(\mathrm{Hb})$ es del $22 \%$ a favor de los nativos de altura ${ }^{(14)}$; el contenido arterial de oxígeno $\left(\mathrm{CaO}_{2}\right)$ es igual o ligeramente mayor (15); la P50 es similar en nativos andinos y residentes de nivel del mar ${ }^{(10)}$; existe mayor concentración del 2,3 difosfoglicerato (2,3 DPG), desplazamiento de la curva de afinidad de la $\mathrm{Hb}$ por el oxígeno hacia la derecha en la zona periférica (efecto Bohr) y desplazamiento de la curva a la izquierda a nivel central (efecto Haldane), fenómenos que estarían incrementados en los nativos de altura; el corazón tiene mayor peso con hipertrofia ventricular derecha, por presentar hipertensión pulmonar sistémica (16); menor gasto cardiaco (GC), menor resistencia arterial en vasos grandes, el cociente $\mathrm{pO}_{2} / \mathrm{FiO}_{2}$ es menor ${ }^{(17)}$.

Bajo la hipótesis que la respuesta ventilatoria es inversamente proporcional a la concentración a la presión parcial de oxígeno inspirado, se han realizado estimaciones con la finalidad de conocer los niveles gasométricos a diferentes altitudes, variando considerablemente conforme se asciende en la altura (18-20). Para estas estimaciones de la presión parcial de oxigeno $\left(\mathrm{pO}_{2}\right)$, se calcula primero la presión parcial de dióxido de carbono $\left(\mathrm{pCO}_{2}\right)$, mediante las fórmulas de Hurtado (18) o Pérez-Padilla (20), $\mathrm{pCO}_{2}=40,4$ - 1,35 (altura en $\mathrm{km}$ ) o $\mathrm{pCO}_{2}=38,3-2,5$ (altura en $\mathrm{km}$ ) respectivamente; luego se calcula la $\mathrm{pAO}_{2}=$ (presión barométrica- $\mathrm{P}$ vapor de agua a $\left.37{ }^{\circ} \mathrm{C}\right) \times\left(0,21-\mathrm{pCO}_{2} / 0,8\right)$ y, finalmente, haciendo uso de la diferencia alveolo-arterial de oxígeno $\left(\mathrm{AaO}_{2}\right)$, descrita como $1 \mathrm{mmHg}$ en el estudio de Hurtado y $6 \mathrm{mmHg}$ en el de Pérez Padilla, estimamos la $\mathrm{pO}_{2}{ }^{(18,20)}$.

Mediante el análisis de gases arteriales podemos medir la función respiratoria, la perfusión tisular e indirectamente el metabolismo celular, cuantificando $\mathrm{pO}_{2}, \mathrm{pCO}_{2}, \mathrm{AaO}_{2}$, $\mathrm{Hb}$, hematocrito (Hto), potencial de hidrogeniones $(\mathrm{pH})$, saturación de oxihemoglobina $\left(\mathrm{SO}_{2}\right), \mathrm{CaO}_{2}$ y el bicarbonato $\left(\mathrm{HCO}_{3}\right)^{(21)}$. Esta información recogida nos permitirá desarrollar nuestros objetivos: determinar los valores gasométricos que tienen los pobladores alto-andinos, compararlos con la población que vive al nivel del mar y con las valores estimados mediante fórmulas para, finalmente comprender de mejor manera la fisiología del habitante bajo estas condiciones ambientales.

\section{MATERIALES Y MÉTODOS}

\section{DISEÑO Y POBLACIÓN DE ESTUDIO}

Estudio transversal analítico para poblaciones que habitan a la misma altitud en el distrito de Santiago de la provincia del Cusco, situada a 3350 m de altitud; presión barométrica $(\mathrm{pB})$ de $505 \mathrm{~mm}$ Hg y presión atmosférica de oxígeno 106,5 mm Hg (Servicio Nacional de Meteorología e Hidrología del Perú- SENAMHI); con una población para el año 2007 de 83721 habitantes, con 43494 entre 20 y 59 años de edad (Instituto Nacional de Estadística e Información-INEI). 


\section{MUESTRA}

La selección fue realizada a través del muestreo por conveniencia de una población voluntaria en el distrito de Santiago de la provincia del Cusco a $3350 \mathrm{~m}$ de altitud, los criterios de inclusión fueron: sujetos que nacieron y residieron sus primeros 20 años en el mismo distrito; no practican deporte más de 60 minutos al día; sin hábito tabáquico; sin antecedentes de patología respiratoria, cardiovascular y hematológica, y que residan cinco años previos al estudio en la misma ciudad. El criterio de exclusión fue: que luego de examen clínico no hayan sido catalogados como "clínicamente sanos", el examen médico fue realizado por un médico neumólogo y tres médicos internistas.

Haciendo uso del programa estadístico EPIDAT 3.1, mediante la estrategia de medias e intervalos de confianza (IC) en un estudio piloto previo, se analizó la desviación estándar y la precisión del error de la muestra en la $\mathrm{pO}_{2}$ y $\mathrm{pCO}_{2}$ con IC $95 \%$ (valor z 1,96), siendo los resultados parciales 115 y 96 sujetos, respectivamente, se estimó necesario una muestra de 110 personas para el trabajo. Inicialmente fueron seleccionados 180 sujetos voluntarios quienes cumplieron los criterios de inclusión y dieron su consentimiento para participar en el estudio, de los cuales 121 no cumplieron los criterios de exclusión a quienes se les realizó la gasometría y, finalmente, fueron 118 las muestras válidas.

\section{PROCEDIMIENTOS}

La recolección de la gasometría se realizó siguiendo el manual de toma de muestras para exámenes de laboratorio de la Universidad de Valparaíso ${ }^{(22)}$, siendo recolección única de muestra, con anestesia local y realizada por los investigadores principales, el análisis de la muestra estuvo a cargo del equipo técnico del Hospital Antonio Lorena de la ciudad del Cusco y fue de manera inmediata a la obtención; el gasómetro utilizado fue ROCHE-OMNI S3 modelo cobas b121 system, se midieron los parámetros de $\mathrm{pO}_{2}, \mathrm{pCO}_{2}, \mathrm{pAO}_{2}, \mathrm{AaO}_{2}, \mathrm{pH}$, $\mathrm{HCO}_{3}, \mathrm{pO}_{2} / \mathrm{FiO}_{2}, \mathrm{AG}, \mathrm{Na}+, \mathrm{K}+, \mathrm{Ca}^{2+}, \mathrm{Cl}-, \mathrm{O} 2 \mathrm{Hb}, \mathrm{Hb}$, Hemoglobina total (tHb), SO2, Hto y $\mathrm{pB}$, calibrado para este estudio.

\section{ANÁLISIS ESTADÍSTICO}

Se realizó un análisis descriptivo de las variables de estudio, utilizando medidas de tendencia central y dispersión, asumiendo la normalidad mediante las pruebas de Kolmogorov-Smirnov, en caso de no seguir una distribución normal, se utilizó la mediana y el rango intercuartílico (RIC); para la comparación de las variables se emplearon las pruebas de asociación t de Student y el análisis de varianza. Se realizaron pruebas de correlación lineal entre las variables gasométricas de interés con la edad y el IMC, para luego realizar regresiones lineales múltiples; se usó el nivel de significación estadístico de $p<0,05$; los valores encontrados se compararon con los calculados mediante fórmulas para la misma altitud. El programa estadístico utilizado fue Stata versión 11.0.

\section{CONSIDERACIONES ÉTICAS}

El trabajo respetó los derechos de los sujetos participantes de acuerdo con los principios éticos y el código de conducta de la American -Psychological Association (2003), y en la declaración de Helsinski II. Se realizó una ficha de consentimiento informado, el cual tuvo que ser firmado para ser incluido al estudio.

\section{RESULTADOS}

De los 118 sujetos de estudio el $57,6 \%$ fueron mujeres; la media global de edad fue 35,39 años; la edad se diferenció en dos grupos, siendo de 20 a 39 años 75 $(63,6 \%)$ y de 40 a 59 años $43(36,4 \%)$. En la Tabla 1 se describe las medias, intervalos de confianza (IC) y distribución por sexo de las variables no gasométricas.

Los resultados de las variables gasométricas fueron: $\mathrm{pH}$ 7,43 mEq/L; $\mathrm{pO}_{2} 61,08 \mathrm{mmHg} ; \mathrm{pCO}_{2} 30,62 \mathrm{mmHg} ; \mathrm{pAO}_{2}$ $62,52 \mathrm{mmHg} ; \mathrm{SO}_{2}$ 91,13 mmHg; Hto 44,2\%; Hb $14,74 \mathrm{mg} /$ dL; $\mathrm{CaO}_{2}$ 18,18 vols/\%; $\mathrm{HCO}_{3} 19,74 \mathrm{mmol} / \mathrm{L} ; \mathrm{pO}_{2} / \mathrm{FiO}_{2}$ 290,79; Anión GAP (AG) 20,9; para todos los casos $p<0,001$, Tabla 2.

Las diferencias por sexo para las variables gasométricas se recogen en la Tabla 3. Del mismo modo, se presentan

Tabla 1. Parámetros no gasométricos calculados por sexo

\begin{tabular}{|c|c|c|c|c|c|c|}
\hline \multirow{2}{*}{ Variable } & \multicolumn{2}{|c|}{ Mujeres } & \multicolumn{2}{|c|}{ Hombres } & \multicolumn{2}{|c|}{ Global } \\
\hline & Media & (IC 95\%) & Media & (IC 95\%) & Media & (IC 95\%) \\
\hline Frecuencia respiratoria (por minuto) & 16,2 & $(16,0-16,4)$ & 16,1 & $(15,9-16,4)$ & 16,2 & $(16,0-16,4)$ \\
\hline Frecuencia cardiaca (por minuto) & 69,1 & $(67,4-70,7)$ & 68,7 & $(66,0-71,4)$ & 68,9 & $(67,4-70,3)$ \\
\hline índice de masa corporal & 24,9 & $(24,1-25,7)$ & 24,8 & $(24,0-25,6)$ & 24,8 & $(24,3-25,4)$ \\
\hline Presión arterial sistólica & 100,4 & $(98,6-102,3)$ & 102,2 & $(99,8-104,6)$ & 101,2 & $(99,7-102,7)$ \\
\hline Presión arterial diastólica & 66,8 & $(65,2-68,3)$ & 67,7 & $(66,2-69,2)$ & 67,2 & $(66,1-68,2)$ \\
\hline
\end{tabular}


Tabla 2. Valores gasométricos calculados en el estudio

\begin{tabular}{|c|c|c|c|}
\hline Variable & Media & $\begin{array}{l}\text { Error } \\
\text { estándar }\end{array}$ & (IC 95\%) \\
\hline $\mathrm{pH} \mathrm{mEq} / \mathrm{L}^{*}$ & 7,4 & 0,0 & $(7,4-7,4)$ \\
\hline $\mathrm{pO}_{2} \mathrm{mmHg}^{*}$ & 61,1 & 0,5 & $(60,0-62,1)$ \\
\hline $\mathrm{pCO}_{2} \mathrm{mmHg}^{*}$ & 30,6 & 0,3 & $(30,0-31,2)$ \\
\hline $\mathrm{pAO}_{2} \mathrm{mmHg}^{*}$ & 62,5 & 0,4 & $(61,7-63,4)$ \\
\hline $\mathrm{SO}_{2} \%$ * & 91,1 & 0,2 & $(90,7-91,6)$ \\
\hline $\mathrm{AaO}_{2}$ & - & - & $(0,0-2,8)$ \\
\hline Hto \%* & 44,2 & 0,4 & $(43,5-44,9)$ \\
\hline $\mathrm{Hb} \mathrm{mg} / \mathrm{dL}^{*}$ & 14,7 & 0,1 & $(14,5-14,9)$ \\
\hline $\mathrm{CaO}_{2}$ vols \%* & 18,2 & 0,2 & $(17,9-18,5)$ \\
\hline $\mathrm{HCO}_{3} \mathrm{mmol} / \mathrm{L}^{*}$ & 19,7 & 0,0 & $(19,4-20,1)$ \\
\hline $\mathrm{pO}_{2} / \mathrm{FiO}_{2}^{*}$ & 290,8 & 247 & $(285,9-295,7)$ \\
\hline Anión GAP* & 20,9 & 0,3 & $(20,4-21,6)$ \\
\hline
\end{tabular}

* $p<0,001{ }^{* *}$ Distribución asimétrica; $\mathrm{pH}$ : potencial de hidrogeniones; $\mathrm{pO}_{2}$ : presión arterial de oxígeno; $\mathrm{pCO}_{2}$ : presión arterial de dióxido de carbono; $\mathrm{pAO}_{2}$ : presión alveolar de oxígeno; $\mathrm{SO}_{2}$ : saturación de oxígeno; $\mathrm{AaO}_{2}$ : diferencia alveolo arterial de oxígeno; Hto: hematocrito; $\mathrm{Hb}$ : hemoglobina; $\mathrm{CaO}_{2}$ : contenido arterial de oxígeno; $\mathrm{HCO}_{3}$ : bicarbonato; $\mathrm{pO}_{2} / \mathrm{FiO}_{2}$ : cociente presión arterial de oxígeno/fracción inspiratoria de oxígeno; AG: anión GAP

Tabla 3. Valores gasométricos según sexo

\begin{tabular}{|c|c|c|c|c|}
\hline \multirow{2}{*}{ Variables } & \multicolumn{2}{|c|}{ Mujeres } & \multicolumn{2}{|c|}{ Hombres } \\
\hline & Media & (IC 95\%) & Media & (IC 95\%) \\
\hline $\mathrm{pO}_{2}$ & 61,4 & $(59,9-62,8)$ & 60,7 & $(59,2-62,2)$ \\
\hline $\mathrm{pCO}_{2}{ }^{*}$ & 29,9 & $(29,1-30,6)$ & 31,6 & $(30,6-32,6)$ \\
\hline $\mathrm{pAO}_{2}{ }^{*}$ & 63,2 & $(62,1-64,3)$ & 61,5 & $(60,2-62,8)$ \\
\hline $\mathrm{SO}_{2}$ & 91,3 & $(90,7-91,9)$ & 90,9 & $(90,2-91,5)$ \\
\hline Hto* & 43,2 & $(42,3-44$ & 45,6 & $(44,3-46,9)$ \\
\hline $\mathrm{Hb}^{*}$ & 14,4 & $(14,1-14,7)$ & 15,2 & $(14,8-15,6)$ \\
\hline $\mathrm{CaO}_{2}{ }^{*}$ & 17,8 & $(17,4-18,1)$ & 18,7 & $(18,2-18,1)$ \\
\hline $\mathrm{HCO}_{3}{ }^{*}$ & 19,4 & $(19,00-20,81)$ & 20,2 & $(19,7-20,7)$ \\
\hline $\mathrm{AaO}_{2}$ & 0,4 & $(\sim 0,0-3,4)$ & 0,0 & $(0,0-1,1)$ \\
\hline $\mathrm{pO}_{2} / \mathrm{FiO}_{2}$ & 292,2 & $(285,8-299,9)$ & 288,9 & $(285,9-295,9)$ \\
\hline AG & 21 & $(20,3-21,7)$ & 20,9 & $(19,9-21,9)$ \\
\hline
\end{tabular}

Tabla 4. Valores gasométricos según grupos de edad

\begin{tabular}{lccccc}
\hline \multirow{2}{*}{ Variable } & \multicolumn{2}{c}{ 20-39 años } & & \multicolumn{2}{c}{ 40-59 años } \\
\cline { 2 - 3 } \cline { 5 - 6 } & Media & (IC 95\%) & & Media & (IC 95\%) \\
\hline $\mathrm{pO}_{2}{ }^{* *}$ & 62,4 & $(61,1-63,7)$ & & 58,8 & $(57,2-60,4)$ \\
$\mathrm{pCO}_{2}{ }^{*}$ & 30,1 & $(29,3-30,9)$ & & 31,5 & $(30,6-32,3)$ \\
$\mathrm{pAO}_{2}{ }^{*}$ & 63,4 & $(62,3-64,5)$ & & 61,0 & $(59,8-62,1)$ \\
$\mathrm{SO}_{2}{ }^{*}$ & 91,6 & $(91,1-92,2)$ & & 90,3 & $(89,5-90,9)$ \\
$\mathrm{Hto}^{*}$ & 44,1 & $(43,1-45,1)$ & & 44,4 & $(43,1-45,7)$ \\
$\mathrm{Hb}^{*}$ & 14,7 & $(14,4-15,0)$ & & 14,8 & $(14,4-15,2)$ \\
$\mathrm{CaO}_{2}$ & 18,2 & $(17,9-18,6)$ & & 18,1 & $(17,5-18,6)$ \\
$\mathrm{HCO}_{3}{ }^{* * *}$ & 19,5 & $(19,1-19,9)$ & & 20,1 & $(19,6-20,6)$ \\
$\mathrm{AaO}_{2}{ }^{*}$ & $0,0^{\mathrm{a}}$ & $(0,0-1,4)^{\mathrm{a}}$ & & $1,0^{\mathrm{a}}$ & $(0,0-4,5)^{\mathrm{a}}$ \\
$\mathrm{pO}_{2} / \mathrm{FiO}_{2}{ }^{* *}$ & 297,0 & $(290,9-303,1)$ & 280,0 & $(272,5-287,5)$ \\
$\mathrm{AG}$ & 21,2 & $(20,4-21,9)$ & 20,7 & $(19,7-21,8)$ \\
\hline
\end{tabular}

a Mediana y RIC; ${ }^{*} p<0,05 ;{ }^{* *} p<0,001 ;{ }^{* * *} p=0,076 ; \mathrm{pH}$ : potencial de hidrogeniones; $\mathrm{pO}_{2}$ : presión arterial de oxígeno; $\mathrm{pCO}_{2}$ : presión arterial de dióxido de carbono; $\mathrm{pAO}_{2}$ : presión alveolar de oxígeno; $\mathrm{SO}_{2}$ : saturación de oxígeno; $\mathrm{AaO}_{2}$ : diferencia alveolo arterial de oxígeno; $\mathrm{Hto}$ hematocrito; $\mathrm{Hb}$ : hemoglobina; $\mathrm{CaO}_{2}$ : contenido arterial de oxígeno; $\mathrm{HCO}_{3}$ : bicarbonato; $\mathrm{pO}_{2} / \mathrm{FiO}_{2}$ : cociente presión arterial de oxígeno/ fracción inspiratoria de oxígeno; AG: anión GAP

los cálculos de los valores gasométricos, diferenciados por grupo de edad, entre 20-39 y 40-59 años, donde existen diferencias significativas $\left({ }^{*} p<0,05{ }^{* *} p<0,001\right)$ como se observa en la Tabla 4.

Existe correlación lineal positiva $(r)$ entre las variables de interés y edad-IMC en $\left(\mathrm{pCO}_{2}=0,237 ; \mathrm{HCO}_{3}=0,195\right)$ $p<0,05$ y correlación negativa en $\left(\mathrm{pO}_{2}=-0,362 ; \mathrm{pAO}_{2}=-\right.$ 0,$\left.308 ; \mathrm{SO}_{2}=-0,346 ; \quad \mathrm{pO}_{2} / \mathrm{FiO}_{2}=-0,361\right) \quad p<0,05 ;$ como existe correlación entre edad e IMC, se valoró mediante regresión lineal múltiple la confusión por parte del IMC, que se describe en la Tabla $5 \mathrm{y}$, puede observarse que a partir de los 20 años de edad, por cada año que cumple una persona, disminuye la $\mathrm{pO}_{2}$ en $0,122 \mathrm{mmHg}$, la $\mathrm{pAO}_{2}$ en $0,08 \mathrm{mmHg}$, la $\mathrm{SO}_{2}$ en $0,05 \%$, el índice $\mathrm{pO}_{2} / \mathrm{FiO}_{2}$ en $0,571 \mathrm{mmHg}$, y por cada año que cumple una persona luego de los 20 años se incrementa la $\mathrm{pCO}_{2}$ en 0,056 mmHg; para todos $p<0,05$; el $\mathrm{HCO}_{3}$ pierde significancia

Tabla 5. Regresión lineal entre valores gasométricos y edad, ajustados por Índice de Masa Corporal

\begin{tabular}{lcccccc}
\hline Variable & $\begin{array}{c}\text { Diferencia de la } \\
\text { media }\end{array}$ & $\begin{array}{c}\text { Diferencia de la Media } \\
\text { ajustada por IMC }\end{array}$ & IC 95\% & $p$ & $\mathbf{R}^{2}$ \\
\hline $\mathrm{pO}_{2}{ }^{*}$ & $-0,173$ & $-0,122$ & $-0,03$ & $-0,21$ & 0,007 & 0,18 \\
$\mathrm{pCO}_{2}{ }^{*}$ & 0,067 & 0,056 & 0,112 & 0 & 0,052 & 0,06 \\
$\mathrm{pAO}_{2}{ }^{*}$ & $-0,119$ & $-0,08$ & $-0,006$ & $-0,153$ & 0,033 & 0,14 \\
$\mathrm{SO}_{2}{ }^{*}$ & $-0,071$ & $-0,05$ & $-0,011$ & $-0,088$ & 0,012 & 0,17 \\
$\mathrm{PaO}_{2} / \mathrm{FiO}_{2}{ }^{*}$ & $-0,819$ & $-0,571$ & $-0,153$ & $-0,99$ & 0,008 & 0,13 \\
$\mathrm{HCO}_{3}$ (NS) & 0,029 & 0,018 & 0,048 & $-0,012$ & 0,237 & 0,06 \\
\hline
\end{tabular}

${ }^{*} p<0,05$; NS: no significativo; $\mathrm{pO}_{2}$ : presión arterial de oxígeno; $\mathrm{pCO}_{2}$ : presión arterial de dióxido de carbono; $\mathrm{pAO}_{2}$ : presión alveolar de oxígeno; $\mathrm{SO}_{2}$ : saturación de oxígeno; $\mathrm{HCO}_{3}$ : bicarbonato; $\mathrm{pO}_{2} / \mathrm{FiO}_{2}$ : cociente presión arterial de oxígeno/fracción inspiratoria de oxígeno; AG: anión GAP 
Tabla 6. Regresión lineal entre valores gasométricos e Índice de Masa Corporal, ajustados por edad

\begin{tabular}{lcccccc}
\hline Variable & $\begin{array}{c}\text { Variación } \\
\text { de la } \\
\text { media }\end{array}$ & $\begin{array}{c}\text { Medias } \\
\text { ajustadas } \\
\text { por edad }\end{array}$ & IC 95\% & $\boldsymbol{p}$ & $\mathbf{R}^{2}$ \\
\hline $\mathrm{pO}_{2}{ }^{*}$ & $-0,704$ & $-0,491$ & $-0,852$ & $-0,131$ & 0,008 & 0,18 \\
$\mathrm{pCO}_{2}$ & 0,207 & 0,109 & $-0,12$ & 0,34 & 0,348 & 0,06 \\
$\left(\mathrm{NS}^{2}{ }^{*}\right.$ & $-0,516$ & $-0,376$ & $-0,676$ & $-0,077$ & 0,014 & 0,14 \\
$\mathrm{pAO}_{2}{ }^{*}$ & $-0,292$ & $-0,206$ & $-0,362$ & $-0,05$ & 0,01 & 0,17 \\
$\mathrm{SO}_{2}{ }^{*}$ & $-3,37$ & $-2,37$ & $-4,08$ & $-0,67$ & 0,007 & 0,18 \\
$\mathrm{PaO}_{2}{ }^{\prime}$ & 0,14 & 0,109 & $-0,01$ & 0,23 & 0,076 & 0,06 \\
$\mathrm{FiO}_{2}{ }^{*}$ & & &
\end{tabular}

* $\mathrm{p}<0,05$; NS: no significativo; $\mathrm{pO}_{2}$ : presión arterial de oxígeno; $\mathrm{pCO}_{2}$. presión arterial de dióxido de carbono; $\mathrm{pAO}_{2}$ : presión alveolar de oxígeno; $\mathrm{SO}_{2}$ : saturación de oxígeno; $\mathrm{HCO}_{3}$ : bicarbonato; $\mathrm{pO}_{2} / \mathrm{FiO}_{2}$ Cociente presión arterial de oxígeno/fracción inspiratoria de oxígeno; AG: Anión GAP

luego de ajustar por IMC $p=0,237$.

En la Tabla 6, se describe la regresión lineal entre los valores gasométricos e IMC ajustados por edad y se observa que, por cada unidad de incremento en el IMC "disminuyen" la $\mathrm{pO}_{2}$ en $0,491 \mathrm{mmHg}$, la $\mathrm{pAO}_{2}$ en $0,376 \mathrm{mmHg}$, la $\mathrm{SO}_{2}$ en $0,206 \%$, el índice $\mathrm{pO}_{2} / \mathrm{FiO}_{2}$ en $2,37 \mathrm{mmHg}$, para todos $p<0,05$; el $\mathrm{HCO}_{3}$ incrementa en $0,109 \mathrm{mmHg}$ con $p=0,076$; la $\mathrm{pCO}_{2}$ pierde fuerza de asociación cuando es ajustada por edad.

En la Tabla 7, se muestran los valores gasométricos comparados entre varios estudios a diferentes altitudes, al hacer el contraste de hipótesis de los resultados hallados $\mathrm{pCO}_{2}=30,62 \mathrm{mmHg}$ y $\mathrm{pAO}_{2}=62,52 \mathrm{mmHg}$, con los resultados inferidos de las fórmulas de Hurtado y Pérez-Padilla, los contrastes favorecen a descartar la hipótesis nula de probabilidad de igualdad $\mathrm{pCO}_{2} 35,88$ mmHg "Hurtado" ( $p<0,001) ; \mathrm{pCO}_{2} 29,92$ mmHg "PérezPadilla" $(p=0,027)$ y para la $\mathrm{pAO}_{2} 51,33 \mathrm{mmHg}$ "Hurtado" $(p<0,001) ; \mathrm{pAO}_{2} 58,78 \mathrm{mmHg}$ "Pérez-Padilla" $(p<0,001)$; del mismo modo, se describen las diferencias con los resultados en otros estudios a altitudes similares.

\section{DISCUSIÓN}

Los valores gasométricos son diferentes en las personas que viven a diferentes altitudes respecto a las que viven a nivel del mar, la $\mathrm{pB}$ del oxígeno cumple un papel importante en el suministro de oxígeno al organismo, conforme se incrementa de altitud sobre el nivel del mar, las presiones dependientes del oxígeno disminuyen progresivamente $^{(2,20,23)}$.

Como consecuencia de la disminución de la $\mathrm{pAO}_{2}$, se encuentra disminución de la $\mathrm{pO}_{2}$, ocasionando adaptaciones fisiológicas a este medioambiente, esta reportado que el incremento de la ventilación basal es el principal mecanismo adaptativo, que es regulado por el arco quimio-reflejo entre receptores carotideos periféricos y el centro de centro respiratorio del tronco encefálico $(3,5,24)$; en este estudio se encontró la FR en rangos normales, efecto que se puede explicar por la hipertrofia de células tipo I en el glomus carotideo, acumulación de dopamina "como principal neurotransmisor depresor del centro respiratorio" y disminución de la sensibilidad de los quimiorreceptores periféricos a la $\mathrm{pO}_{2}{ }^{(6,25)}$; por otro lado en personas altoandinas al estar incrementado el VAC ${ }^{(7)}$ y la capacidad vital forzada (CVF) ${ }^{(26)}$, se puede explicar también por qué la FR se mantiene en rangos similares a los que viven a nivel del mar.

Tabla 7. Contraste de hipótesis de los valores hallados en el presente estudio y los calculados en las fórmulas de Hurtado, Pérez-Padilla para $\mathrm{pCO}_{2}$ y $\mathrm{pAO}_{2}$

\begin{tabular}{|c|c|c|c|c|c|c|c|}
\hline $\begin{array}{c}\text { Valores } \\
\text { gasométricos }\end{array}$ & $\begin{array}{l}\text { Presente } \\
\text { estudio: }\end{array}$ & $\begin{array}{c}\text { Cálculo } \\
\text { mediante la } \\
\text { fórmula de } \\
\text { Hurtado }^{(18)}\end{array}$ & $\begin{array}{c}\text { Cálculo } \\
\text { mediante } \\
\text { la fórmula } \\
\text { de Pérez- } \\
\text { Padilla }^{(20)}\end{array}$ & $\begin{array}{l}\text { Estudio: } \\
\text { Gonzales- } \\
\text { García } \\
\text { Bogotá(29) }^{2}\end{array}$ & $\begin{array}{l}\text { Estudio: Yumpo } \\
\text { Huancayo }\end{array}$ & $\begin{array}{l}\text { Libro "El reto } \\
\text { de vivir en los } \\
\text { Andes"(2) }\end{array}$ & Tietz $^{(30)}$ \\
\hline & $3350 \mathrm{~m}$ & $3350 \mathrm{~m}$ & $3350 \mathrm{~m}$ & $2640 \mathrm{~m}$ & $3249 \mathrm{~m}$ & $3600 \mathrm{~m}$ & “0”m \\
\hline$\overline{\mathrm{pH}} \mathrm{mEq} / \mathrm{l}$ & 7,426 & - & - & 7,42 & 7,452 & - & 7,4 \\
\hline $\mathrm{pO}_{2} \mathrm{mmHg}$ & 61,075 & 45,33 & 57,78 & 63,1 & 66,225 & 52 & 95,5 \\
\hline $\mathrm{pCO}_{2} \mathrm{mmHg}$ & $30,62^{*}$ & $35,88^{*}$ & $29,925^{\star *}$ & 30,3 & 29,16 & 35 & 40 \\
\hline $\mathrm{pAO}_{2} \mathrm{mmHg}$ & $62,519^{*}$ & $51,33^{*}$ & $58,78^{*}$ & 71,8 & - & - & 105 \\
\hline $\mathrm{AaO}_{2} \mathrm{mmHg}$ & $0,0^{\mathrm{a}}$ & 6 & 1 & 8,7 & - & - & 14 \\
\hline $\mathrm{SO}_{2} \%$ & 91,133 & - & - & 92,4 & 93,9 & 83 & 96 \\
\hline $\mathrm{HCO}_{3} \mathrm{mmol} / \mathrm{l}$ & 19,741 & - & - & 20,2 & 20,52 & - & 24,5 \\
\hline $\mathrm{pO}_{2} / \mathrm{FiO}_{2}$ & 290,793 & - & - & 300,476 & 313,357 & 247,619 & 450 \\
\hline
\end{tabular}

a Mediana; ${ }^{*} p<0,001 ;{ }^{* *} p<0,05 ; \mathrm{pH}$ : potencial de hidrogeniones; $\mathrm{pO}_{2}$ : presión arterial de oxígeno; $\mathrm{pCO}_{2}$ : presión arterial de dióxido de carbono; $\mathrm{pAO}$ : presión alveolar de oxígeno; $\mathrm{SO}_{2}$ : saturación de oxígeno; $\mathrm{AaO}_{2}$ : diferencia alveolo arterial de oxígeno; $\mathrm{HCO}_{3}$ : bicarbonato; $\mathrm{pO}_{2} / \mathrm{FiO}_{2}$ : $\mathrm{cociente} \mathrm{presión}$ arterial de oxígeno/fracción inspiratoria de oxígeno. 
Respecto a la $\mathrm{AaO}_{2}$ los valores hallados en estudios pequeños, 0 inferidos por fórmulas para altitudes similares, van desde 6 a $9,8 \mathrm{mmHg}{ }^{(20,27)}$, que difieren a los encontrados en el presente estudio; por los resultados se puede corroborar que la hematosis es diferente en el poblador altoandino, en estos habitantes el capilar venoso tiene $\mathrm{pO}_{2}$ de $35 \mathrm{mmHg}$ y recién al final del recorrido en el capilar pulmonar se logra tener valores de $\mathrm{pO}_{2}$ de $45 \mathrm{mmHg}$, entendiendo por tal motivo que la diferencia de la presión de oxígeno es mínima ${ }^{(9)}$; existe además hipertrofia del ventrículo derecho con incremento de la presión en la arteria pulmonar e hipertrofia de las capilares pulmonares, lo que condicionaría mayor perfusión a nivel de los lobulillos pulmonares (28); por estos hallazgos se puede deducir que el gradiente de la $\mathrm{pO}_{2}$ en altura no es un factor importante para el intercambio de oxígeno en la membrana alveolo-capilar, para lo cual sería necesario plantear otros estudios que expliquen el mecanismo de difusión de oxígeno en la membrana alveolo-capilar.

Si la ventilación basal es normal en las personas del estudio, ¿cuál es la explicación de encontrar valores inferiores de $\mathrm{pCO}_{2}$, sabiendo que la hipoxemia no condiciona hipocapnia? Una de la explicaciones planteadas es que al haber mayor capacidad de difusión del $\mathrm{CO}_{2}$ en la membrana alveolo capilar asociada al incremento del volumen corriente, podría manifestarse en una mayor salida de $\mathrm{CO}_{2}$ en cada espiración normal ${ }^{(6)}$; la otra explicación es que la hipoxemia condicione la vía glucolítica anaerobia incrementando la producción de ácido láctico u otros ácidos no volátiles, con ligera disminución final del bicarbonato, debido al sistema buffer activado producto de la vía glucolítica, condicionando una acidosis metabólica (estado encontrado en el estudio). Por lo tanto, se considera que no es correcto llamar al poblador altoandino como alcalótico respiratorio, porque en estas condiciones son capaces de mantener un equilibrio ácido-base ${ }^{(27)}$.

Finalmente para hacer uso de parámetros gasométricos arteriales, es importante tener en cuenta la altitud sobre el nivel del mar en la que vive el paciente, por el riesgo de clasificación y actuación en ellos, además, es necesario tener en cuenta el envejecimiento y el incremente del IMC; los resultados encontrados pueden ser empleados en poblaciones que habitan a una altitud parecida a la del estudio.
Algunas limitaciones merecen ser consideradas. La selección de la muestra fue realizada por conveniencia, pero si vemos la variabilidad de los resultados en la gasometría, todos excepto la $\mathrm{pAO}_{2}$ tienen distribución simétrica con intervalos de confianza pequeños.

La catalogación como persona sana, fue mediante valoración clínica, la que fue realizada inicialmente por anamnesis de antecedentes patológicos de importancia, seguido del examen clínico general por lo médicos internistas, este médico indicaba la realización de radiografía de tórax y hemograma completo; las personas que luego de la evaluación clínica, lectura de la analítica y radiografía de tórax por el médico neumólogo, fueran normales, se les prosiguió con la toma de la muestra gasométrica.

En conclusión, los parámetros gasométricos varían a diferente altitud en la que viven las personas, por lo tanto, es necesario considerar estas variaciones al momento de tomar decisiones durante la clasificación y tratamiento de pacientes expuestos a estas condiciones. La edad y el IMC son parámetros que influyen en la variación de los parámetros gasométricos; el sexo tiene diferencia estadística más no relevancia clínica; estos resultados pueden ser utilizados para poblaciones que habitan en condiciones de altitud similar

Agradecimientos: a Oscar Niño de Guzmán, médico neumólogo del Hospital Antonio Lorena-Cusco; Fredy Daza Valer, médico internista del Hospital Adolfo Guevara Velasco EsSalud-Cusco; Yemis Cáceres Peñalva, médico internistaintensivista del Hospital Adolfo Guevara Velaso EsSaludCusco, quienes apoyaron en la realización de los exámenes clínicos de los sujetos de estudio.

Máxima Lizán-García, jefa del Servicio de Medicina Preventiva del CHUA; Antonio Vázquez Molinero, médico residente del Servicio de Medicina Preventiva y Salud Pública del CHUA, por su ayuda en la redacción y aspectos metodológicos.

Contribuciones de autoría: CPV, JHQ y LCT participaron en la concepción del estudio, recolección de datos, análisis e interpretación de datos, redacción y revisión crítica del artículo.

Conflictos de interés: los autores declaran no tener conflictos de interés.

Fuentes de financiamiento: autofinanciado 


\section{REFERENCIAS BIBLIOGRÁFICAS}

1. Hultgren H. High altitude medicine. Stanford, CA: Hultgren Publishing, 1997.

2. Monge CC, León-Velarde F. El reto fisiológico de vivir en los Andes. Lima: Instituto Francés de Estudios Andinos; 2003.

3. Frisancho AR. Human growth and pulmonary function of a high altitude Peruvian Quechua population. Hum Biol. 1969 Sep;41(3):365-79.

4. Greksa LP, Spielvogel H, Caceres E. Total lung capacity in young highlanders of Aymara ancestry. Am J Phys Anthropol. 1994 Aug;94(4):477-86.

5. Frisancho AR. Developmental adaptation: where we go from here. Am J Hum Biol. 2009 SepOct;21(5):694-703. doi: 10.1002/ ajhb.20891.

6. Lahiri S, Rozanov C, Cherniack NS. Altered structure and function of the carotid body at high altitude and associated chemoreflexes. High Alt Med Biol. 2000 Spring;1(1):63-74.

7. Hurtado A. The influence of high altitude on physiology. CIBA Foundation Symposium. High Altitude Physiology: Cardiac and Respiratory Aspects. London: Longman Group; 1971.

8. Burton MD, Kazemi H. Neurotransmitters in central respiratory control. Respir Physiol. 2000 Sep;122(2-3):111-21.

9. Gozal D, Gaultier C. Evolving concepts of the maturation of central pathways underlying the hypoxic ventilatory response. Am J Respir Crit Care Med. 2001 Jul 15;164(2):325-9.

10. Fatemian M, Robbins PA. Selected contribution: chemoreflex responses to $\mathrm{CO} 2$ before and after an 8-h exposure to hypoxia in humans. J Appl Physiol (1985). 2001 Apr;90(4):1607-14.

11. Fatemian M, Gamboa A, LéonVelarde F, Rivera-Ch M, Palacios J-A, Robbins PA. Selected contribution: Ventilatory response to $\mathrm{CO} 2$ in highaltitude natives and patients with chronic mountain sickness. J Appl Physiol. 2003 Mar;94(3):1279-87.

12. Reynafarje C. Iron metabolism during and after altitude exposure in man and in adapted animals (camelids). Fed Proc. 1966 Jul-Aug;25(4):1240-2.
13. Monge CC. Regulación de la concentración de hemoglobina en la policitemia de altura: modelo matemático. Bull Inst Fr Etud Andines. 1990;19(2):455-467.

14. León-Velarde F, Monge CC, Vidal A, Carcagno M, Criscuolo M, Bozzini CE. Serum immunoreactive erythropoietin in high altitude natives with and without excessive erythrocytosis. Exp Hematol. 1991 May;19(4):257-60.

15. Villafuerte FC, Cárdenas R, Monge-C C. Optimal hemoglobin concentration and high altitude: a theoretical approach for Andean men at rest. J Appl Physiol. 2004 May;96(5):1581-8.

16. Arias-Stella J, Recavarren S. Right ventricular hypertrophy in native children living at high altitude. Am J Pathol. 1962 Jul;41:55-64.

17. Pérez-Padilla JR. La altitud modifica la relación entre la $\mathrm{PaO} 2 / \mathrm{FiO} 2$ y el cortocircuito: impacto en la valoración de la lesión pulmonar aguda. Arch Bronconeumol. 2004 Oct;40(10):45962.

18. Hurtado A. Handbook of Physiology. Adaptation to the Environment. Washington, DC: Am Physiol Soc 1964.

19. García JCV, Padilla RP. Valores gasométricos estimados para las principales poblaciones y sitios a mayor altitud en México. Rev Inst Nal Enf Resp Mex. 2000;13(1):6-13.

20. Pérez Padilla JR, Vázquez García JC. Estimación de los valores gasométricos en diferentes alturas sobre el nivel del mar en México. Rev Invest Clin. 2000;52(2):148-55.

21. Rodriguéz-Roisín R, García-Navarro AA, Rincón FB, Pere Casan C, Tordera MP, Agudo LS, et al. Gasometría arterial [Internet]. Barcelona: Separ; 1998 [citado el 10 de agosto de 2013]. Disponible en: http://www.hispasante. be/documentacion/guias/enfer/ Gasometria_arterial.pdf

22. Navarro C. Manual de técnicas de toma de muestra para exámenes de laboratorio [Internet]. Valparaíso: Universidad de Valparaíso: 2005 [citado el 10 de agosto de 2013]. Disponible en: http://prontus.uv.cl/pubacademica/ pubprofesores/l/publandmancecilia/ site/artic/20070119/asocfile/manual exs.pdf
23. Hinojosa-Campero WE. Gasometría arterial y adaptación en la altura. Rev Med Cient Luz Vida. 2011;2(1):39-45.

24. Frisancho AR. Developmental adaptation to high altitude hypoxia. Int J Biometeorol. 1977 Jun;21(2):13546.

25. Pequignot JM, Cottet-Emard JM, Dalmaz Y, Peyrin L. Dopamine and norepinephrine dynamics in rat carotid body during long-term hypoxia. J Auton Nerv Syst. 1987 Nov;21(1):914.

26. Valenzuela Bejarano, MA. Medición de la capacidad vital forzada por espirometría en habitantes adultos naturales de Junín (4105 msnm) y su utilidad en la práctica [Internet]. Lima: UNMSM; 2003 [citado el 11 de setiembre de 2013]. Disponible en: http://sisbib.unmsm.edu.pe/ bibvirtual/monografias/Salud/ valenzuela_bm/contenido.htm.

27. Yumpo Castañeda D. Estudio de valores de referencia de gases arteriales en pobladores de altura. Enfermedades del Tórax. 2002;45:40-2.

28. Rupert JL, Hochachka PW. Genetic approaches to understanding human adaptation to altitude in the Andes. J Exp Biol. 2001 Sep;204(Pt 18):315160.

29. González-García M, Barrero M, Maldonado D. Limitación a la tolerancia al ejercicio en pacientes con EPOC a la altura de Bogotá (2640 $\mathrm{m})$. Patrón respiratorio y gasometría arterial en reposo y en ejercicio pico. Arch Bronconeumol. 2004;40(2):5461.

30. Burtis CA, Ashwood ER, Bruns DE. Tietz textbook of clinical chemistry and molecular diagnostics. 5th ed. Philadelphia: Elsevier Health Sciences; 2012.

Correspondencia: César Pereira Victorio Dirección: Calle Hellin 35 P-07, Albacete, España

Teléfono: +34645487613

Correo electrónico:pereira.victorio.cj@gmail.com 